\title{
Performance Analysis of Voice and Video Conference over DiffServ and MPLS
}

\author{
Sheriff Aboul ${ }^{*}$, Hesham Elbadawy ${ }^{\dagger}$, Abdel Hamid Gaafar ${ }^{\ddagger}$
}

\begin{abstract}
Multiprotocol Label Switching (MPLS) and Differentiated Services (DiffServ) play a vital role for minimizing the congestion by efficient load balancing and management of the network resources used to support Quality of Service (QoS). The lower network delay and efficient forwarding mechanism enhancing the speed of packet transfer make it more suitable for implementing real-time applications such as Voice and Video Conferencing. This paper evaluates the performance measures such as Packet Delay Variation (PDV) and end-to-end delay when applying some case studies comprising numbers of routers serving different number of clients. Both (PDV) and (End-to-End Delay) are determined using combination of Open Shortest Path First (OSPF), DiffServ and MPLS protocols. The results may lead to the measures of selection of the appropriate protocol.
\end{abstract}

Keywords: DiffServ, MPLS, QoS

\section{Introduction}

Many organizations developers are searching now to find the best quality of service (QoS) to send different real time applications over the Internet (i.e. voice and video conferencing). The real time applications provide QoS in terms of delay, packet loss and throughput.

The IETF suggested many service mechanisms and schemes to achieve QoS requirements, the most popular QoS models are IntServe Integrated Service (IntServe), DiffServ and MPLS [1].

In order to provide QoS for more demanding types of applications (e.g., voice, multimedia), a network must satisfy two necessary conditions.

The first condition is that bandwidth must be guaranteed for an application under various circumstances, including congestion and failures.

The second condition is that as an application flow traverses the network, it must receive the appropriate class-based treatment, including scheduling and packet discarding. It is necessary to satisfy both of these conditions in order to achieve the hard QoS guarantees that are required by service providers and their customers. These two concepts meet in the DiffServaware MPLS Traffic Engineering (DS-TE) framework.

Arab Academy of Science and Maritime Technology, sherif_aboul@cs.mti.edu.eg

National Telecommunication Institute, Cairo, Egypt, heshamelmahdy@iee.org

Arab Academy of Science and Maritime Technology, abdelhamid.gaafar@aast.edu 


\section{Modeling of DiffServ}

The DiffServ architecture defined Classes of Service (CoS), called Aggregates, and QoS resource management functions with node-based, or Per-Hop, operation. The CoS definitions include a Behavior Aggregate (BA) which has specific requirements for scheduling and packet discarding, and an Ordered Aggregate (OA) which performs classification based on scheduling requirements only, and may include several drop precedence values. The node behavior definitions correspond to the CoS definitions. A Per Hop Behavior (PHB) is offered to a BA, whereas a PHB Scheduling Class (PSC) serves an OA; PHB mechanisms include scheduling and packet discarding, whereas PSC only concerns scheduling. Fourteen PHBs have been defined, including one for EF (Expedited Forwarding [2]), twelve for AF (Assured Forwarding [3]), and one for Default, or Best Effort, PHB. The twelve AF PHBs are divided into four PSCs, and each of the AF PSCs consists of three sub-behaviors related to different packet discarding treatment [4].

The DiffServ model is based on redefining the meaning of the 8-bit ToS field in the IP header. The original ToS definition was not widely implemented, and now the field is split into the 6bit DSCP (DiffServ Code Point [5]) value and the 2-bit Explicit Congestion Notification $(\mathrm{ECN})$ part, as shown in Figure 1 below.

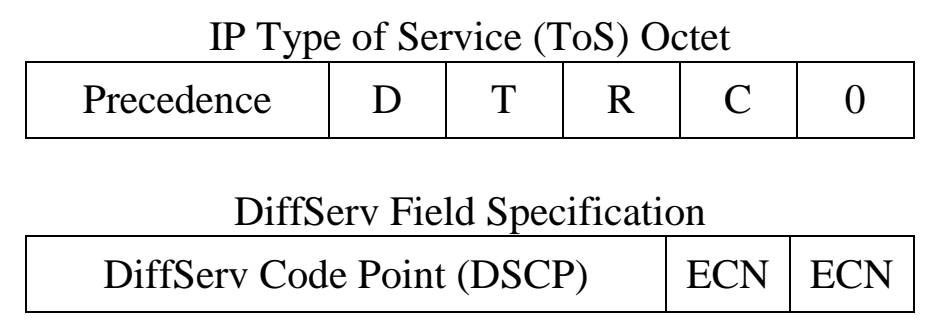

Fig. 1. Relationship between ToS and DiffServ / ECN

\section{MPLS Protocol}

The Multiprotocol Label Switching (MPLS) provides high performance packet control and forwarding mechanism for routing the packets in the data networks which means providing Quality of Service (QoS) and TE.

MPLS is an advanced high-speed packet forwarding scheme, which takes an approach introducing a connection-oriented mechanism into connectionless IP networks [6] [7].

MPLS combines the intelligence of routing with the performance of switching [8]. Its main feature is to attach a short fixed-label to the packets that enter into MPLS domain. Label is placed between Layer 2 (Data Link Layer) and Layer 3 (Network Layer) of the packet to form Layer 2.5 label switched network on layer 2 switching functionality without layer 3 IP routing [2] [9] [10].

The MPLS "Shim Header" has a total length of 32 bits; 20 bits for Label, 3 bits for Experimental (EXP), 1 bit for Bottom of Stack and 8 bits for Time to Live (TTL), as shown in Figure 2 below.

Packets in the MPLS network are forwarded based on these labels. This leads to overcome the limitations like excessive delays and high packet loss of IP networks during routing of packets. 


\begin{tabular}{|c|c|c|c|c|c|c|}
\hline $\begin{array}{c}\text { L2 } \\
\text { Header }\end{array}$ & $\begin{array}{c}\text { Label } \\
\text { (unstructured) }\end{array}$ & Exp & S & TTL & $\begin{array}{c}\text { IP } \\
\text { Header }\end{array}$ & $\begin{array}{c}\text { IP } \\
\text { Data }\end{array}$ \\
\hline
\end{tabular}

Fig. 2. MPLS “shim" header

The main function of IP is to send the data from the source to destination. Data is constructed as a series of packets. All the packets are routed through a chain of routers and multiple networks to reach the destination.

In the Internet, router takes independent decision on each incoming packet. When a packet reaches a router, it forwards the packet to the next hop depending on the destination address present in the packet header. The process of forwarding the packets by the routers is done until the packet reaches the destination.

In the MPLS domain, the MPLS path is known as label switched path (LSP), LSP starts at head-end router (ingress router or label edge router LER) and ends at tail-end router (egress router or LER) [8] [11]. Only two routers (the ingress and egress routers) are responsible for creating (LSP). Core or Label switch routers (LSR) in MPLS network will only forward packets based on labels transmitted through a pre-established LSP [12] [11].

\section{Network Design and Modeling}

OPNET Modeler is applied in performing and analysis of the work presented in this paper. The main feature of OPNET is that it provides various real-life network configuration capabilities that make the simulation environment close to reality [10].

The simulations consist of two groups of scenarios with different network topology:

A) Group A of 5 routers: which can send the three real time applications, namely: voice, video-conference and FTP to each of the following cases:

1) Scenario 1: One client.

2) Scenario 2: Five clients.

3) Scenario 3: Ten clients.

B) Group B of 10 routers: which can send the three real time applications, namely: voice, video-conference and FTP to each of the following cases:

4) Scenario 4: One client.

5) Scenario 5: Five clients.

6) Scenario 6: Ten clients.

The OPNET modeler is applied on the six aforementioned scenarios in order to determine the values of the Packet Delay Variation (PDV) and the (End-to-End Delay) which are supposed to be the measures of selection of the appropriate protocols. Both (PDV) and (End-to-End Delay) are determined using the following protocols:

- OSPF.

- OSPF + MPLS.

- OSPF + DiffServ.

- OSPF + MPLS + DiffServ. 
In addition, this study may lead to know the influence of number of routers and/or number of clients on the value levels of (PDV) and (End-to-End Delay) which in its turn may lead to unexpected limitations of use of the used protocols.

Samples of these groups and scenarios are illustrated in the following figures:

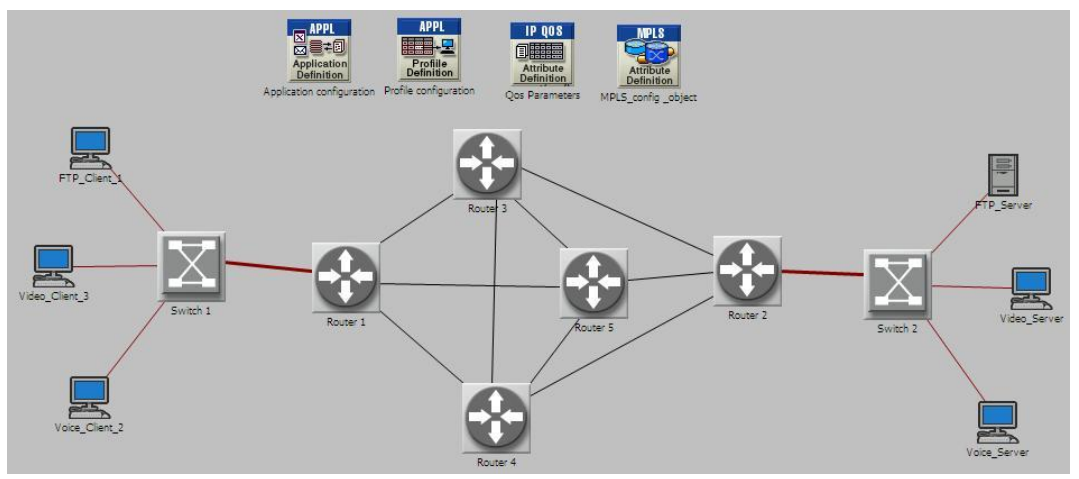

Fig. 3. Five Nodes / One Client Topology

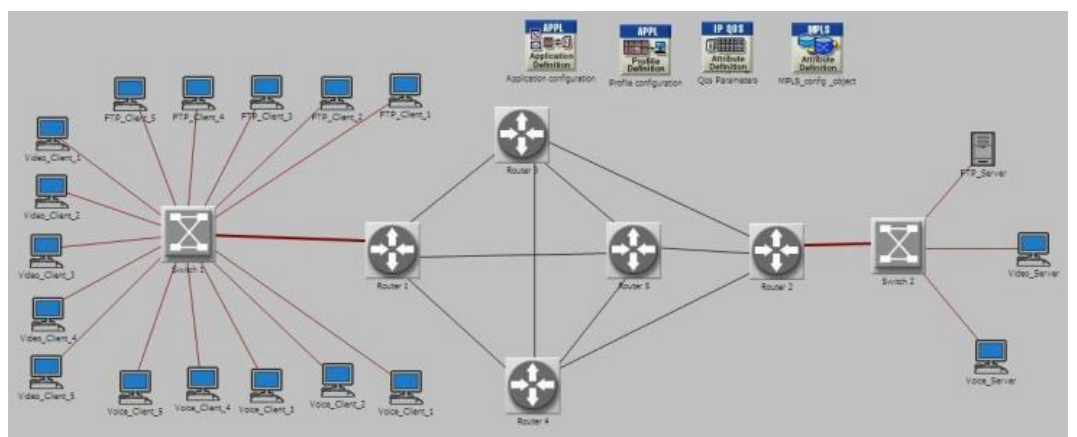

Fig. 4. Five Nodes / Five Clients Topology

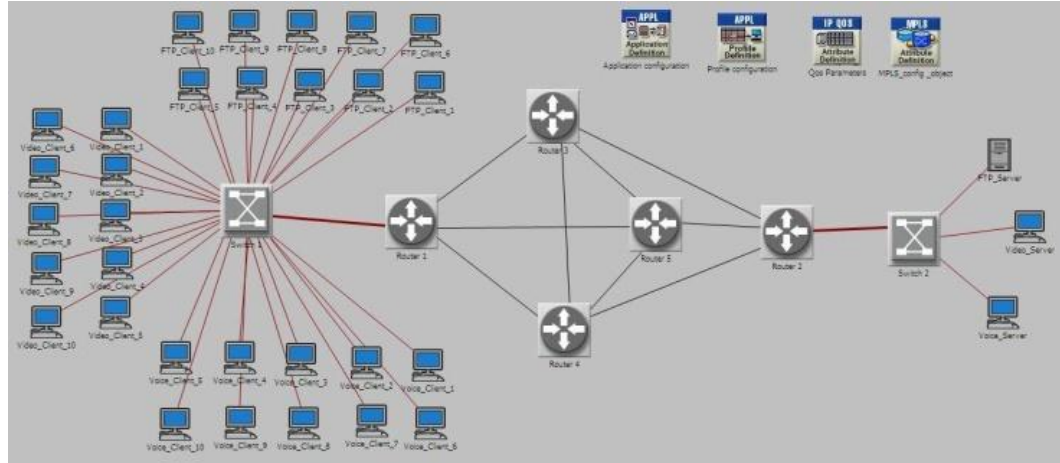

Fig. 5. Five Nodes / Ten Clients Topology 


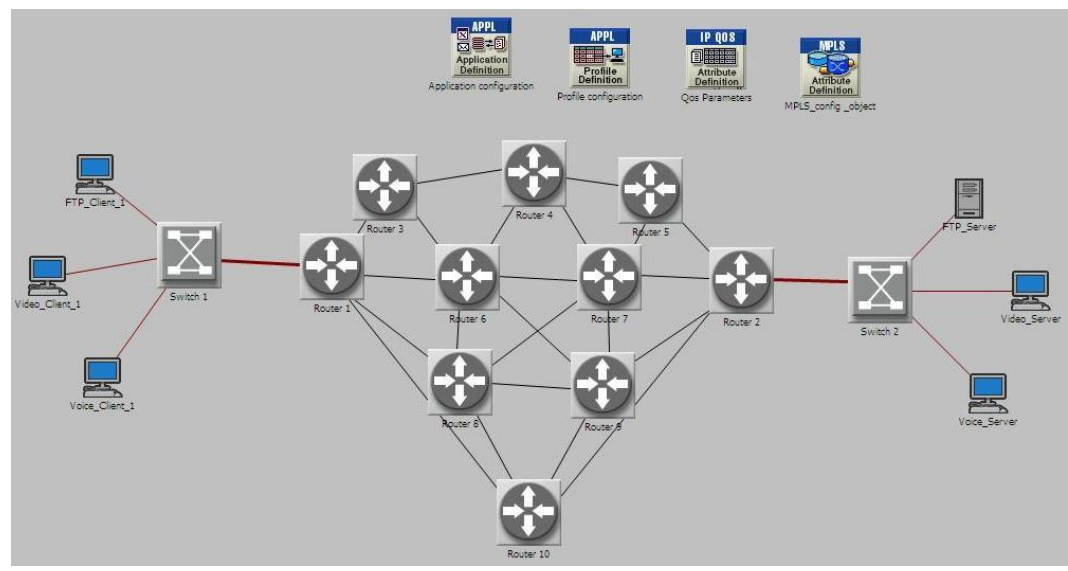

Fig. 6. Ten Nodes / One Client Topology

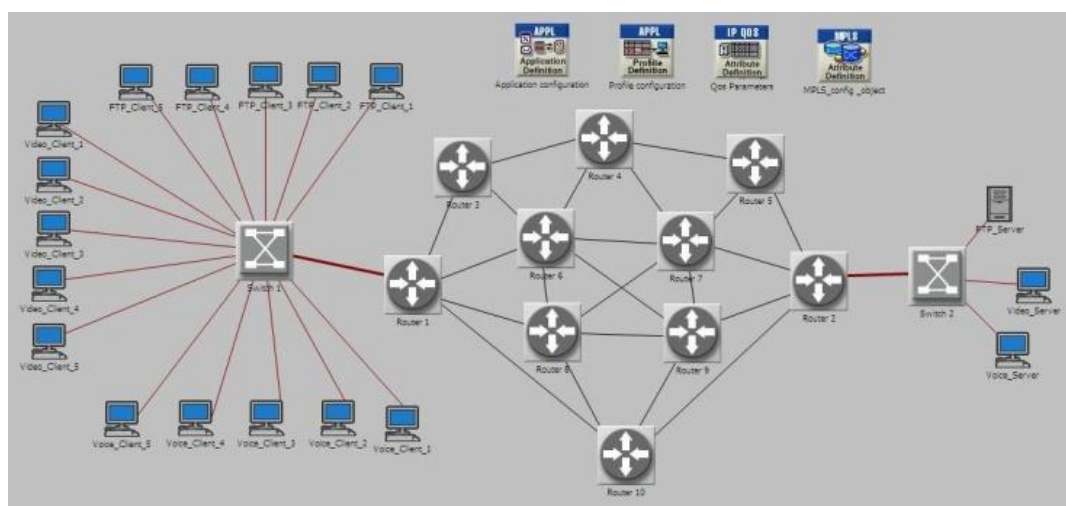

Fig. 7. Ten Nodes / Five Clients Topology

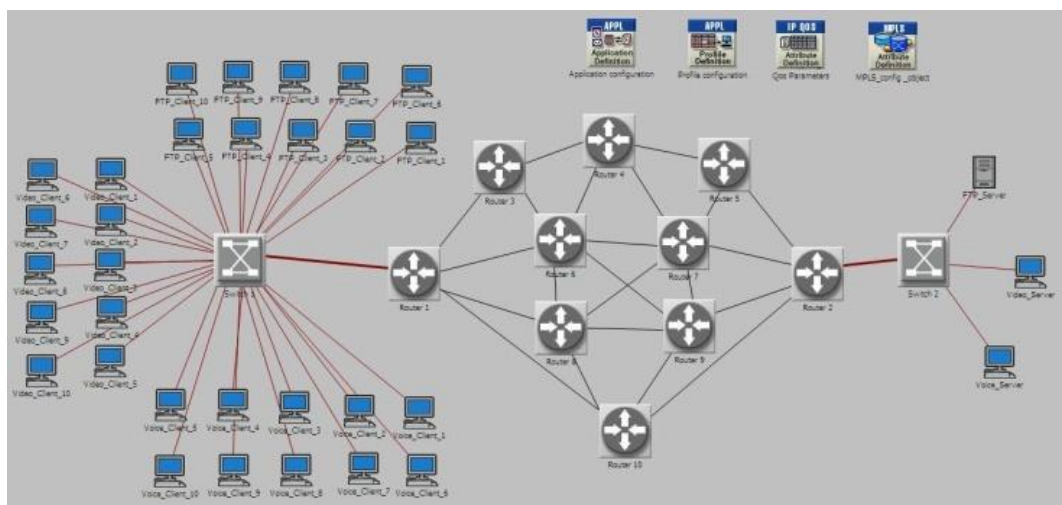

Fig. 8. Ten Nodes / Ten Clients Topology

\section{Results and Analysis}

\section{A) Voice Traffic:}

Applying OPNET modeler to the aforementioned topologies shown in figures (3 to 8) using simulation time of 500 (s), the PDV and End-to-End delays are determined for voice. An example of the simulation results of End-to-End delay in case of 5 Nodes and 5 Clients is shown in figure 9 below. 


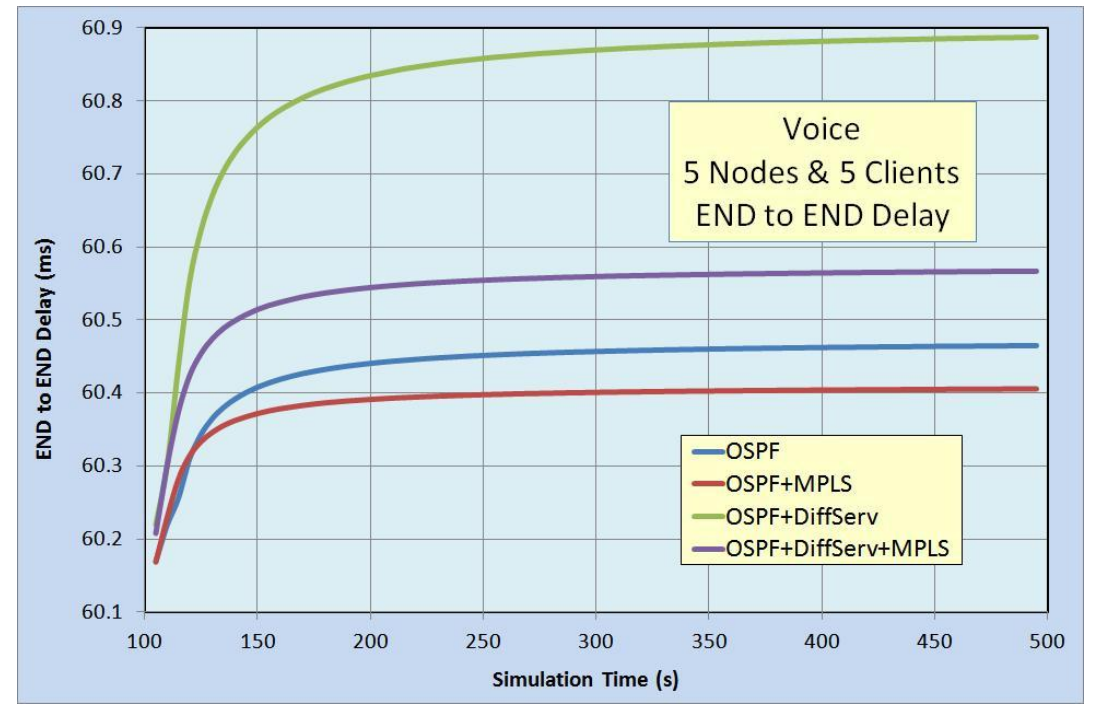

Fig. 9. Example of Voice (End-to-End) Delay Simulation Results (5 Nodes \& 5 Clients)

The values of each simulation parameter at the end of simulation (i.e) at time 500 (s) is used as the comparison parameter for each protocol.

Hereafter, the whole results of Voice PDV and End-to-End delay are presented in figures (10 to 13) for all topologies in the form of column diagrams for the matter of comparison when using different protocols.

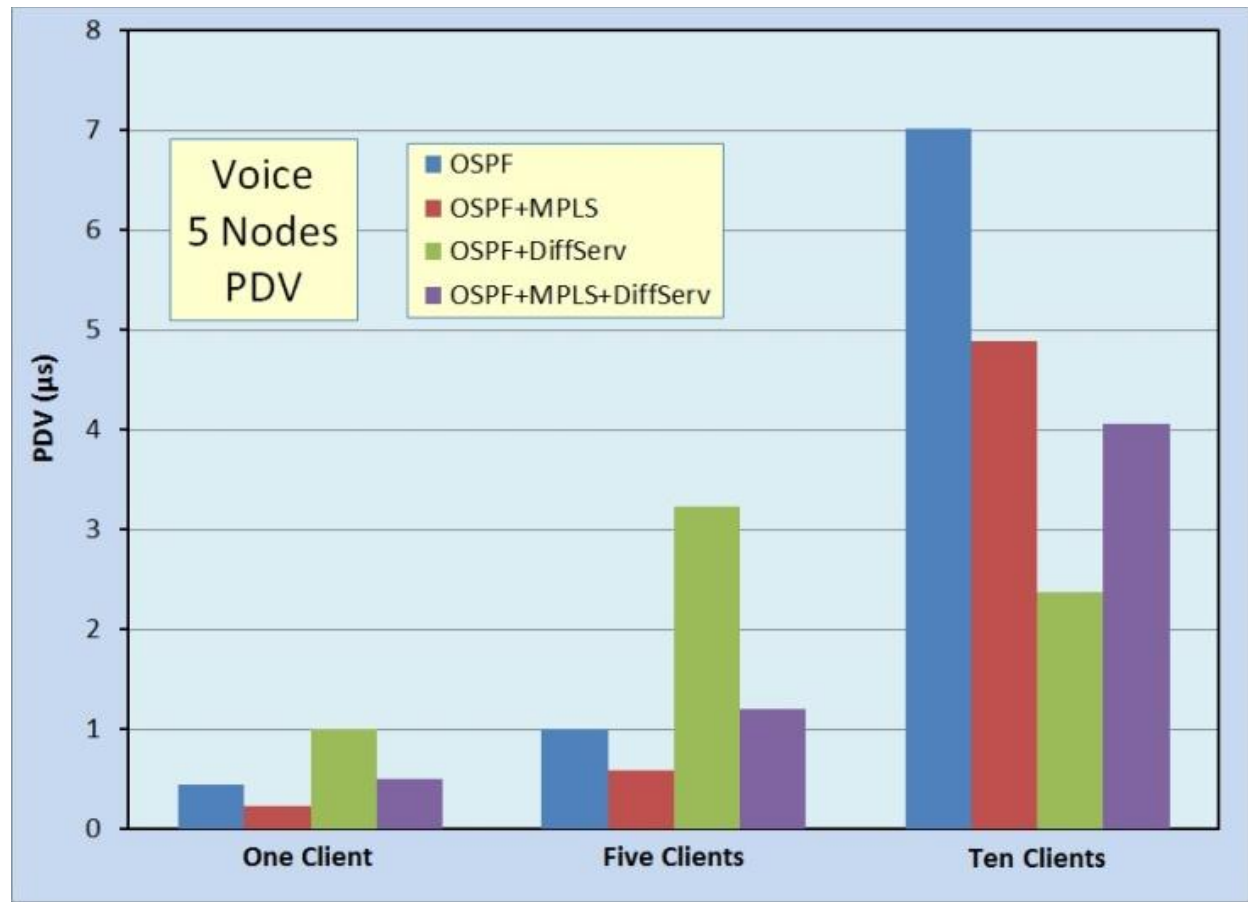

Fig. 10. Voice (PDV) Results for 5 Nodes 


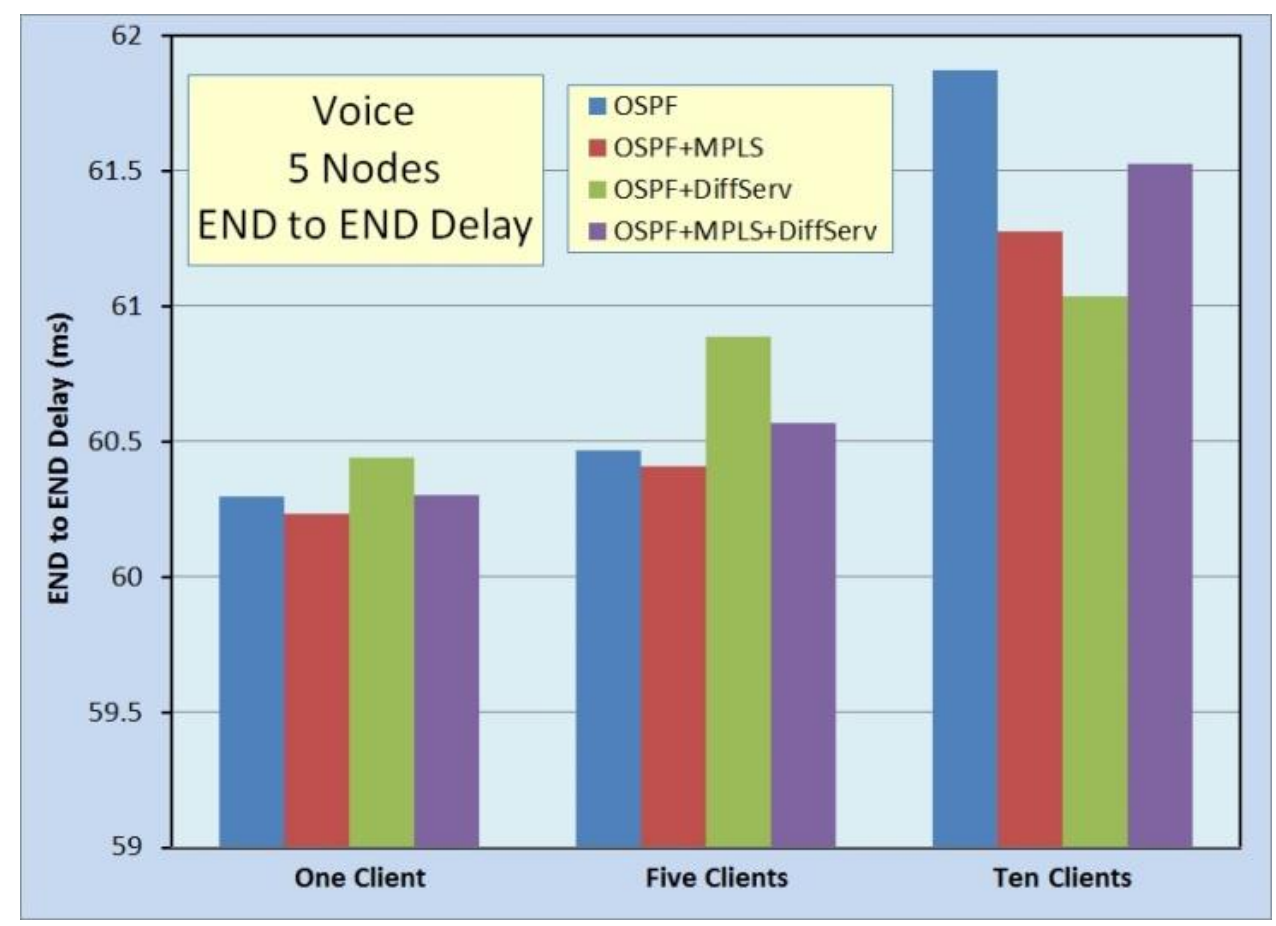

Fig. 11. Voice (End-to-End) Delay Results for 5 Nodes

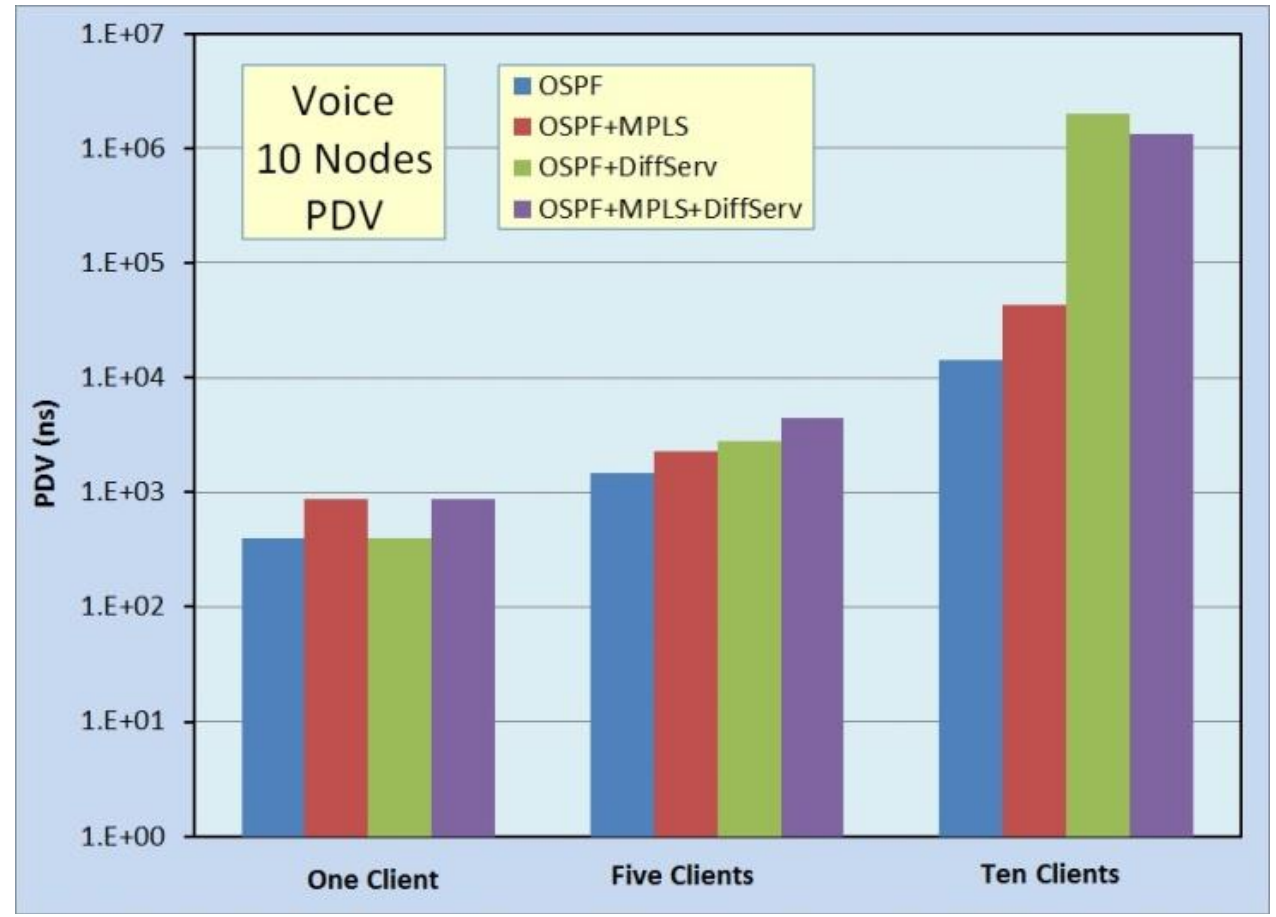

Fig. 12. Voice (PDV) Results for 10 Nodes 


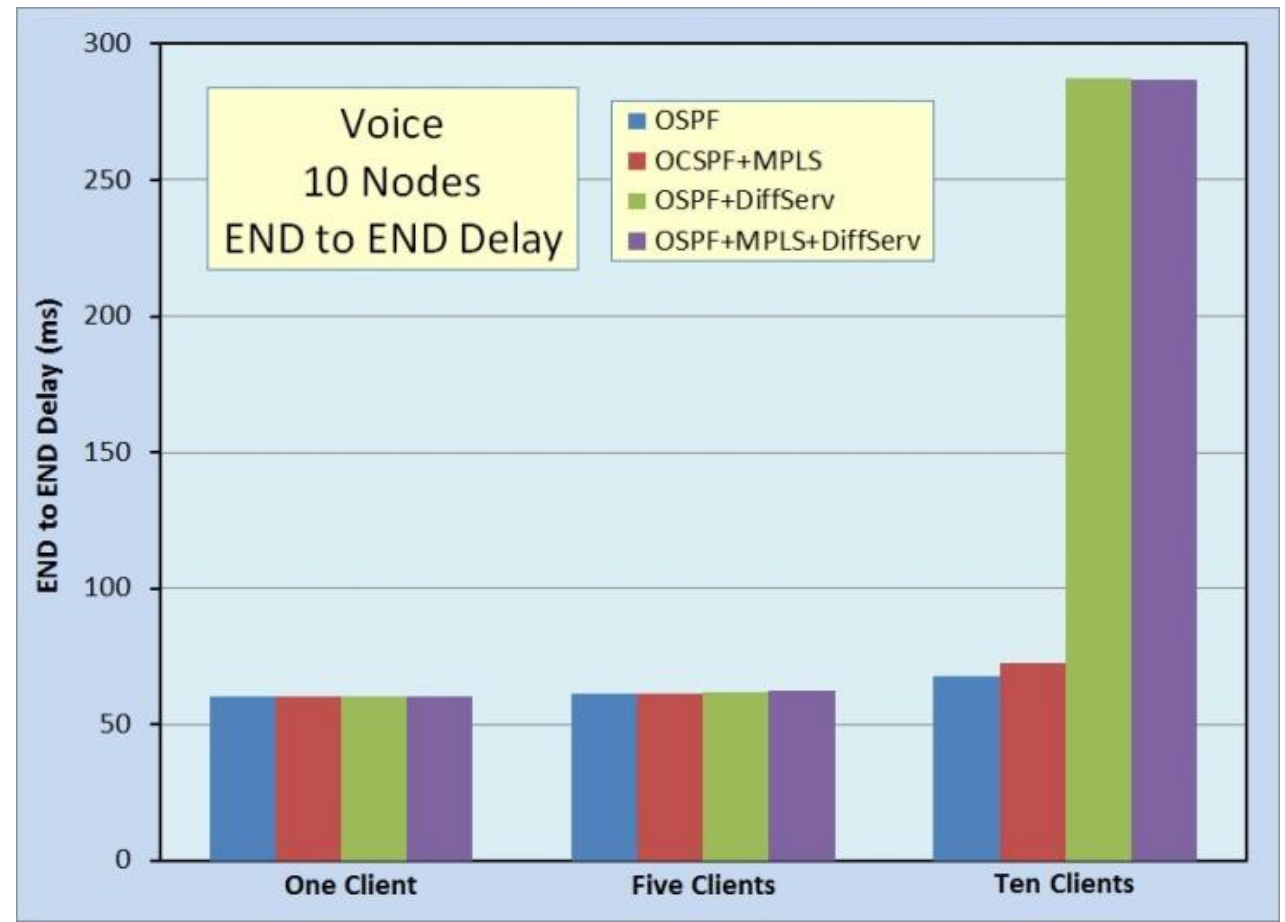

Fig. 13. Voice (End-to-End) Delay Results for 10 Nodes

\section{B) Video Conference Traffic:}

Applying OPNET modeler to the aforementioned topologies shown in figures (3 to 8) using simulation time of 500 (s), the PDV and End-to-End delays are determined for video conferencing. An example of the simulation results of End-to-End delay in case of 5 Nodes and 5 Clients is shown in figure 14 below.

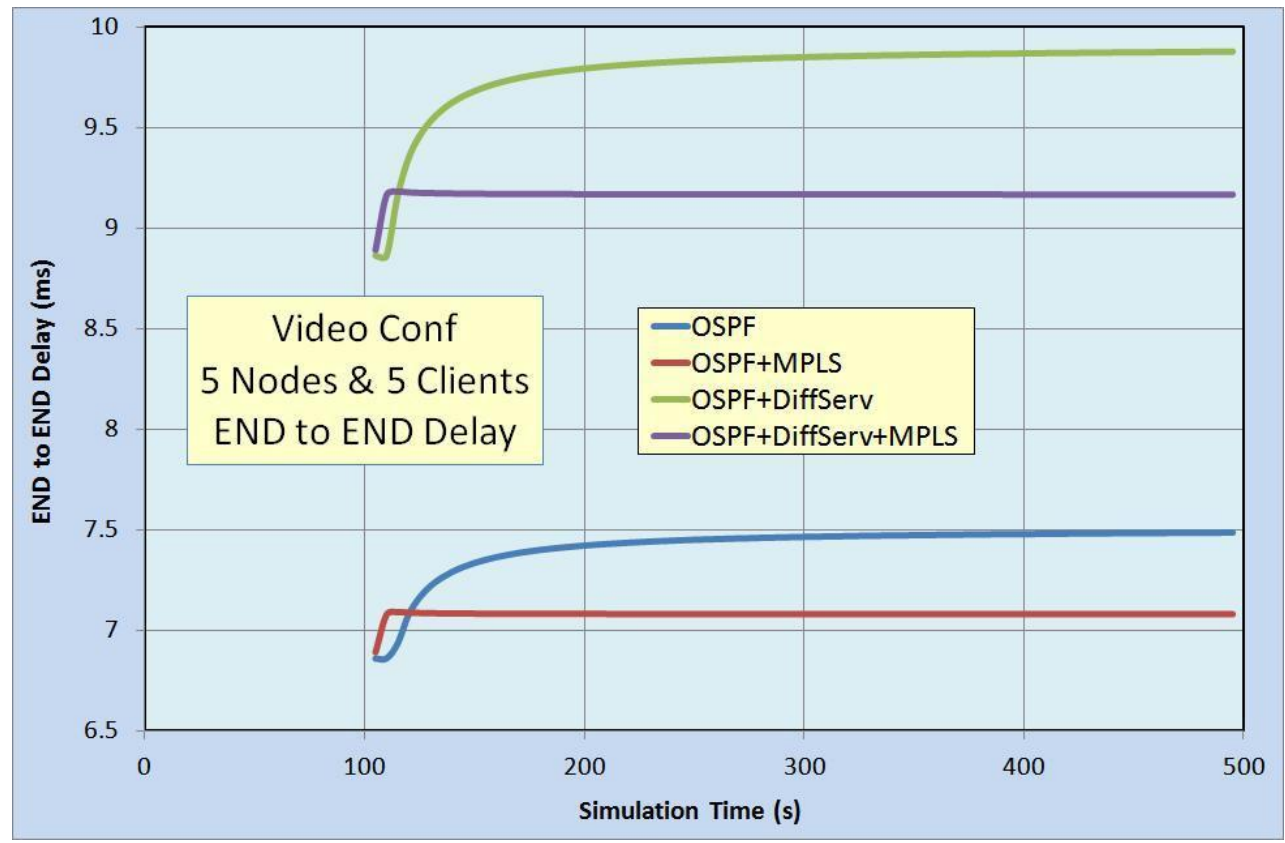

Fig. 14. Example of Video Conferencing (End-to-End) Delay Simulation Results (5 Nodes \& 5 Clients) 
Hereafter, the whole results of Video Conferencing PDV an End-to-End delay are presented in figures (15 to 18) for all topologies in the form of column diagrams for the matter of comparison when using different protocols. The values of each simulation parameter at time 500 (s) is used as the comparison parameter for each protocol.

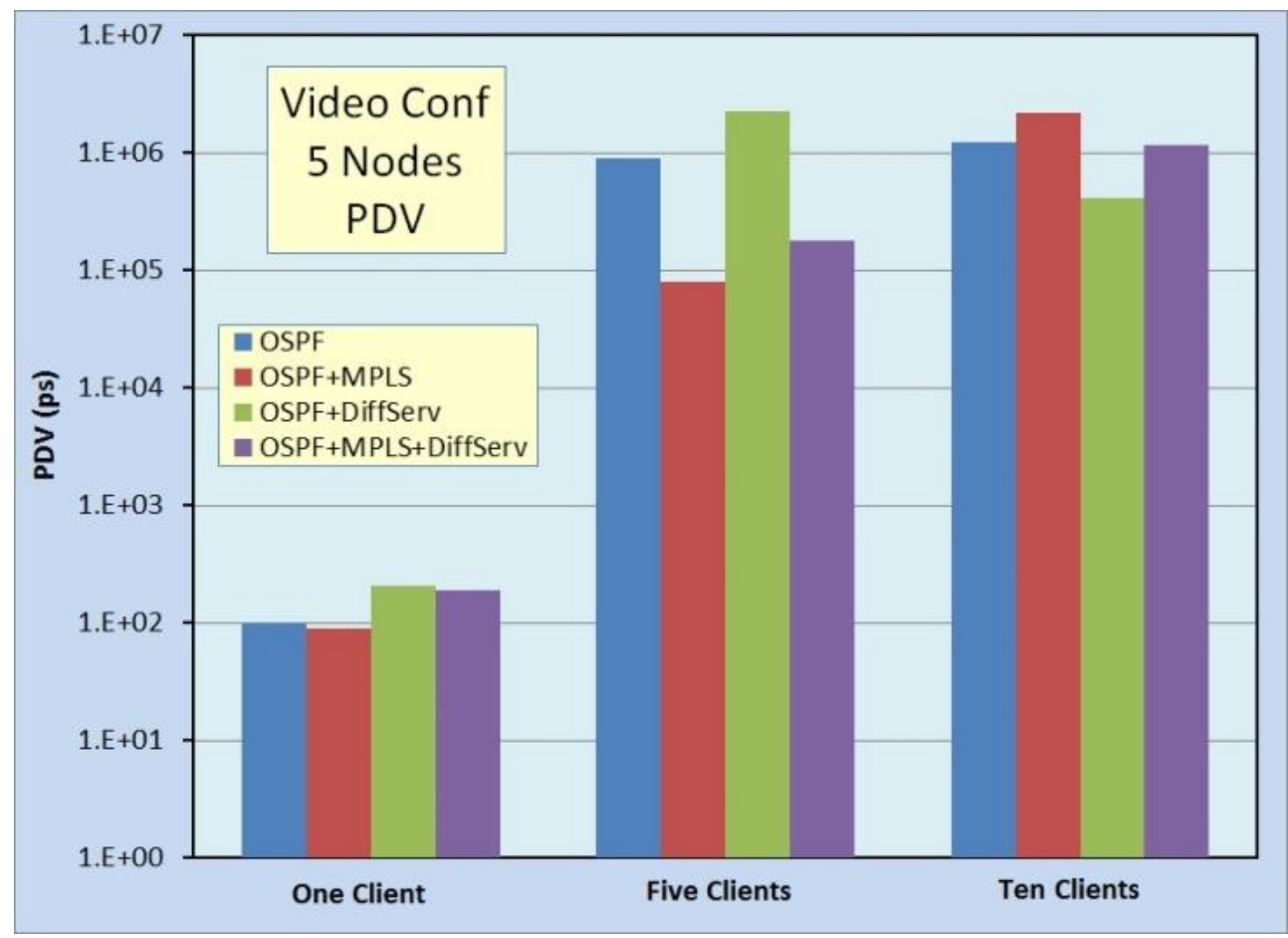

Fig. 15. Video Conferencing (PDV)

Results for 5 Nodes

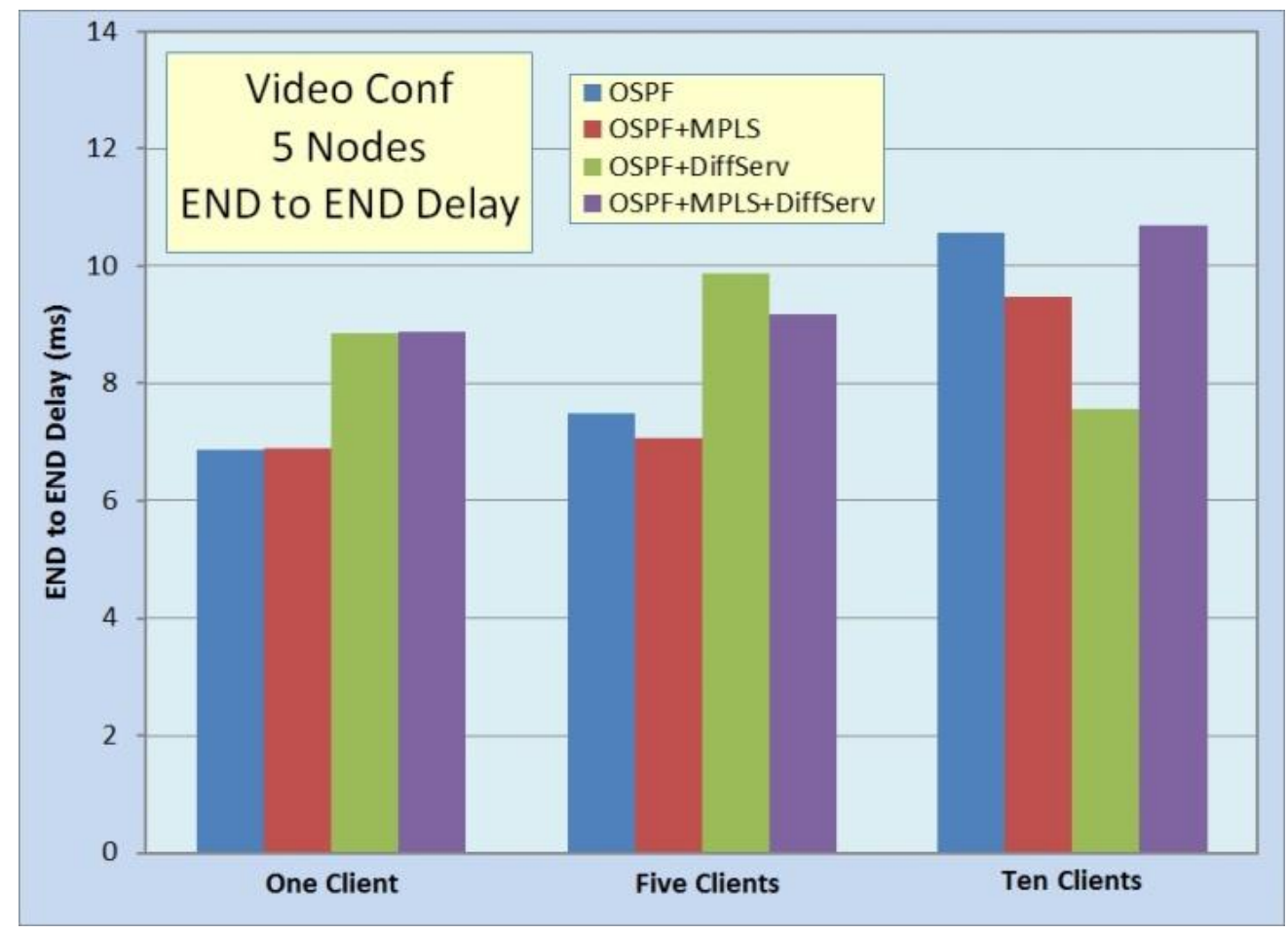

Fig. 16. Video Conferencing (End-to-End) Delay Results for 5 Nodes 


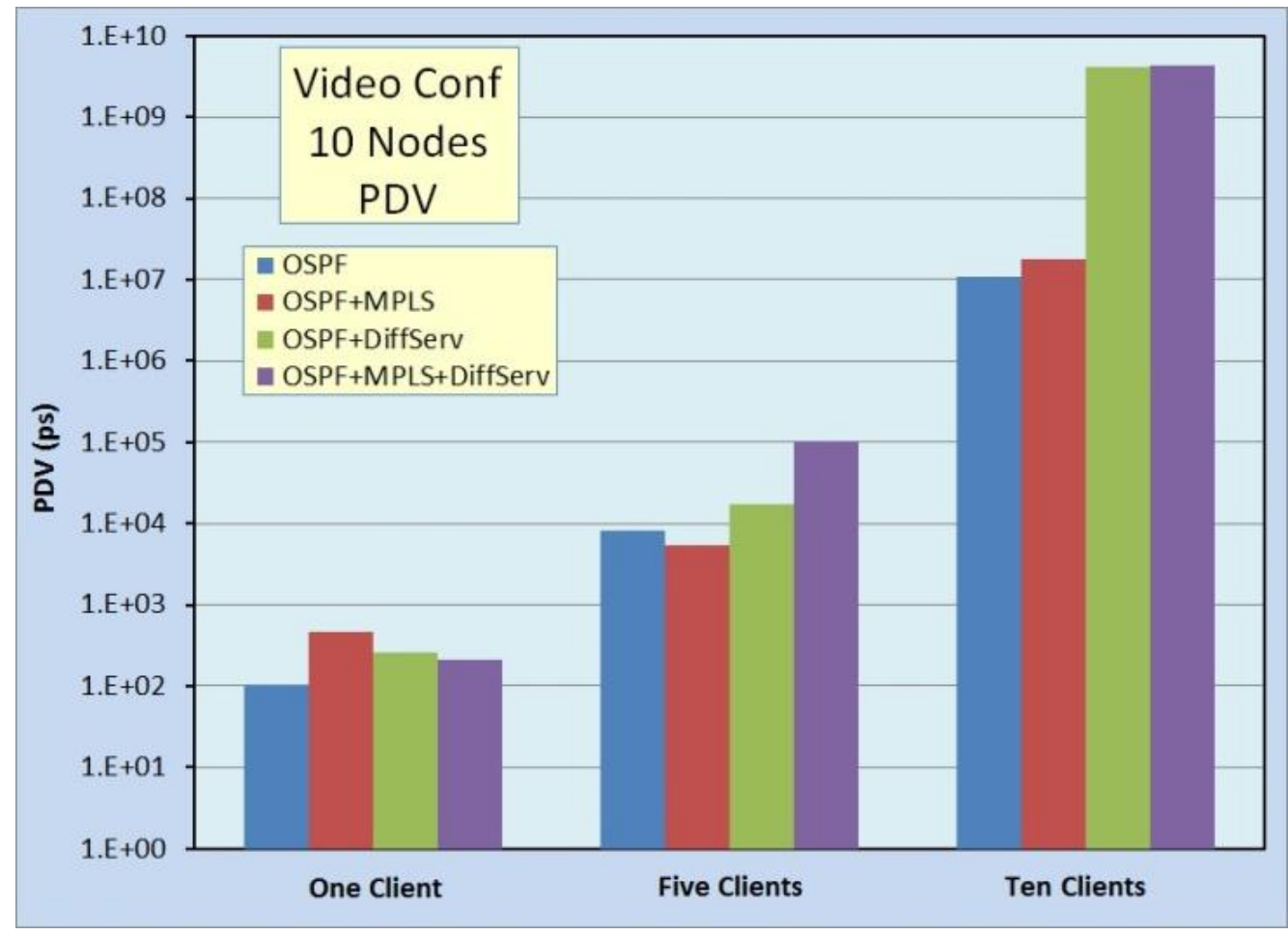

Fig. 17. Video Conferencing (PDV)

Results for 10 Nodes

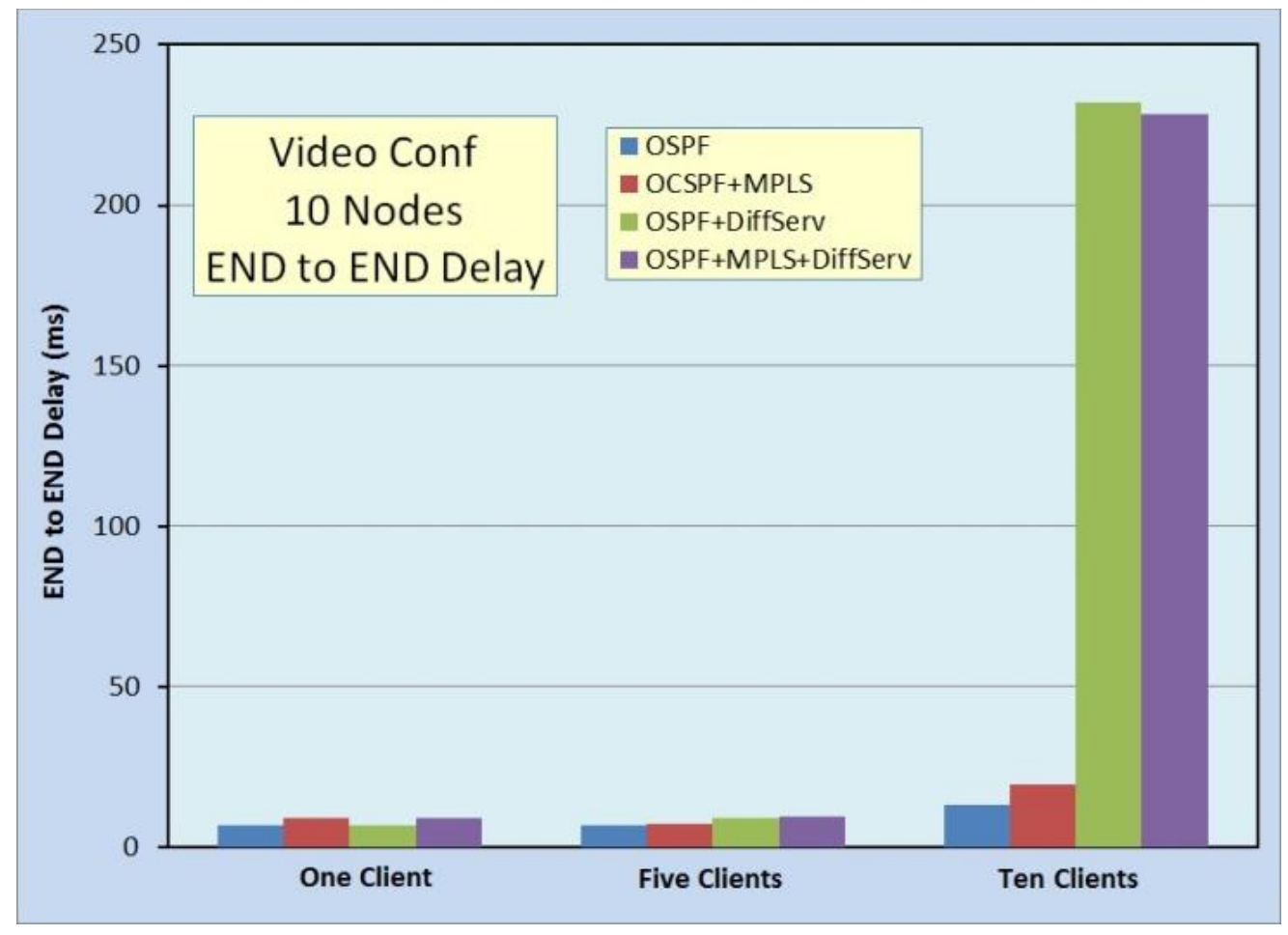

Fig. 18. Video Conferencing (End-to-End) Delay

Results for 10 Nodes 


\section{Conclusion and Future Work}

The evaluation of some performance measures are the stem of this work. These measures such as delay variation and end-to-end delay are applied when studying some case studies comprising various groups of routers serving different numbers of clients. Both (PDV) and (End-to-End Delay) are determined using combination of OSPF, DiffServ and MPLS protocols.

These case studies are held on five and ten nodes serving three different groups of clients (one, five and ten clients) both for voice and video conferencing. The obtained results show that the exploitation of MPLS protocol for Voice improves (layer 3) protocols in low, medium and heavy number of clients. Whereas in video conferencing MPLS protocol improves layer 3 only in low and medium number of clients.

In addition, the application of DiffServ protocol improves layer 3 only in heavy number of clients. Moreover, the combination of applying MPLS and DiffServe protocols is better than the use of DiffServe alone in low and medium number of clients.

Generally, the current work proves that the performance of QoS protocols (MPLS, DiffServe and MPLS/DiffServe) decreases by the increase of number of nodes. As a final conclusion, the performance of QoS protocols may also decrease by the increase of number of paths between nodes. This point merits extra research as a future work.

\section{References}

[1] Ji-Feng Chiu, Zuo-Po Huang, Chi-Wen Lo, Wen-Shyang Hwang and Ce-Kuen Shieh, Supporting End-to-End QoS in DiffServ/MPLS Networks, IEEE Telecommunications, 2003. ICT 2003. 10th International Conference on, 2003.

[2] Er. Sourabh Jain, RGPV Bhopal, Bhopal, "Performance Analysis of Voice over Multiprotocol Label Switching Communication Networks with Traffic Engineering", International Journal of Advanced Research in Computer Science and Software Engineering, Volume 2, Issue 7,July 2012.

[3] J Heinanen, F. Baker, W. Weiss, and J Wroclawski, "Assured forwarding PHB group," RFC 2597, June 1999.

[4] Victoria Fineberg, Consultant, "QoS Support in MPLS Networks", Frame Relay Forum, MPLS/Frame Relay Alliance White Paper, May 2003.

[5] K. Nichols, S. Blake, F. Baker and D. Black, "Definition of the Differentiated Services Field (DS Field) in the IPv4 and IPv6 Headers," RFC 2474, Dec. 1998.

[6] Li Zhenyu and Zhang Zhongzhao, Study on the Class-Based Admission Control Scheme for DiffServ in MPLS Networks, Journal of Systems Engineering and Electronics, Vol. 14, No. 3, 2003, pp.40 - 44.

[7] N.M. Din and N. Fisal, Dynamic resource allocation of IP traffic for a DiffServ-MPLS interface using fuzzy logic, IEEE Communications, 2003. APCC 2003. The 9th AsiaPacific Conference on, Sept. 2003, pp.339-343.

[8] Hasanein Hasan, John Cosmas and Zaharias Zaharis, Creating and Managing Dynamic MPLS Tunnel by Using SDN Notion, IEEE Telecommunications and Multimedia (TEMU), 2016 International Conference on, 2016.

[9] Dr. Abdul-Bary R. Sulaiman and Omar Kh. Salih Alhafidh, "Performance Analysis of Multimedia Traffic over MPLS Communication Networks with Traffic Engineering", International Journal of Computer Networks and Communication Security, Vol. 2, NO. 3, March 2014,93-101. 
[10] K.Salah and A.Alkhoraidly -An OPNET-based simulation approach for deploying VoIP\|, INTERNATIONAL JOURNAL OF NETWORK MANAGEMENT Int. J. Network Mgmt 2006; 16: 159-183.

[11] D. Adami, C. Callegari, S. Giordano, F. Mustacchio, M. Pagano and F. Vitucci, Signalling Protocols in DiffServ-aware MPLS Networks: Design and Implementation of RSVP-TE Network Simulator, GLOBECOM '05. IEEE, 2005, pp.792-796.

[12] S.Veni and Dr.G.M.Kadhar Nawaz, A New Approach To Enhance Security In MPLS Network, An International Journal ( ACIJ ), Vol.3, No.3, May 2012, pp.75-80. 